https://doi.org/10.21278/TOF.452019720

ISSN 1333-1124

eISSN 1849-1391

\title{
TECHNIQUES FOR MESH INDEPENDENT DISPLACEMENT RECOVERY IN ELASTIC FINITE ELEMENT SOLUTIONS
}

\begin{abstract}
Summary
In this study, techniques for mesh dependent and independent displacement recovery for an a posteriori error estimation are presented. The error recovery of the field variable is made by fitting a higher order polynomial to the displacement over a mesh independent patch (support domain) using the moving least square (MLS) interpolation procedure. The mesh dependent recovery procedure is based on the recovery of the displacement over an element patch that consists of all elements surrounding the element under consideration using the least square (LS) interpolation procedure. The two-dimensional benchmark examples are analysed using linear and quadratic triangular elements to demonstrate the effectiveness and reliability of error estimations. Global and elemental errors of a finite element solution in the energy and $\mathrm{L}_{2}$ norms are calculated directly from the post-processed displacement. The quality of error estimation obtained using the mesh independent displacement recovery technique in terms of convergence properties, effectivity and adaptive meshes under different error norms has been compared with that of the mesh dependent displacement recovery using the MLS interpolation and least square (LS) interpolation procedures. The performance of an adaptive scheme based on a mesh independent error estimator is compared with the adaptive scheme based on a mesh dependent error estimator. The numerical results show that the finite element analysis based on mesh independent recovery is very effective in converging to a predefined accuracy in a solution with a significantly smaller number of degrees of freedom.
\end{abstract}

Key words: $\quad$ error; effectivity; error norm; recovery techniques; convergence; least square techniques.

\section{Introduction}

The finite element method has become an effective tool for numerical analysis in the engineering field. In recent years, increased attention has been paid to overcome deficiencies of the finite element method and to improve the performance of the finite element analysis. Chen et al. [1] presented the development taking place in the area of meshless methods in the last two decades, which were developed to overcome drawbacks of the finite element method. A procedure to automatically improve a finite element solution using interpolation covers was propounded by Kim and Bathe [2] and the cover order was established through an error indicator. Li et al. [3] proposed an isogeometric meshfree moving least squares method for the adaptive analysis of static and quasi-static crack propagation in thin-shell structures with a 
higher convergence rate and computational efficiency. The adaptivity of the mesh refinement was achieved by utilizing a gradient-based error estimator to identify the meshes that need to be refined by adding linear reproducing points. Cortis et al. [4] developed a boundary conditions imposition method for dual applicability, i.e. for the standard finite element method as well as for the material point method (MPM) to model boundaries of any inclination in which the problem domain boundary does not coincide with background grid element edges. Liu et al. [5] developed a coupling technique for combining the waveletGalerkin method with the finite element method and established its accuracy and stability for 2D and 3D elasticity problems. Dekker et al. [6] proposed an extended finite element model (XFEM) in an adaptive environment to capture the fatigue crack propagation and crack growth retardation under mixed-mode loading and overloading. A stress-displacement mixed least-squares finite element formulation was proposed by Igelbüscher et al. [7] for elasticplastic material behaviour. Lee et al. [8] analysed hyperelastic bodies with compressible and nearly incompressible neo-Hookean behaviour using the smoothed finite element method (SFEM). The smoothed strains and smoothed deformation gradients were evaluated on subdomains selected by either edge information (edge-based S-FEM, ES-FEM) or nodal information (node-based S-FEM, NS-FEM). Jarak et al. [9] presented meshless methods based on the mixed Meshless Local Petrov Galerkin approach used for solving linear fourthorder differential equations. The method was successfully adapted for an application in gradient elasticity obtaining accurate results even with a low order of meshless approximation functions. The finite element method yielded approximate solutions for the problems and significant errors may creep in the computed solution due to elemental and discretization approximations. The state of the art in different error estimation techniques developed to get a practical finite element solution for linear, non-linear and transient problem analyses was presented by Gratsch and Bathe [10]. The discretization error i.e. the manner of domain discretization, manifested as discontinuities or jumps in the components of stress or strain between elements and such errors were introduced in the problem solution when the displacement interpolation polynomial did not accurately represent the behaviour of the continuum. The discretization error may be reduced by changing the location of mesh nodes or by increasing the mesh/node density. The most popular error estimation techniques, namely the post-processing type [11], are based on the difference between the values of postprocessed recovered, more accurate displacement gradients (or alternatively displacement) and those given by the finite element solution as a measure of the local error. The pointwise definitions are not only difficult to estimate but also become meaningless at singularities. Therefore, an alternative scale measure in the energy norm, representing the error in the rate of energy dissipation, is preferable. Finite element solutions are the best approximations in terms of energy norm [12]. However, the error norms of the displacements, i.e., the basic finite element solution, such as the $\mathrm{L}_{2}$ norm, representing straightforward physical meaning, show many superconvergence properties of finite elements [13]. The main advantage of using the post-processed type error estimation is that it gives not only global but also element error estimates. Further, it can yield information on pointwise error estimation. The post-processing type estimator, i.e. ZZ error estimator [11], has proved to be economical and effective both in evaluating error simply and as an estimator preliminary to the adaptive process [14]. The superconvergent patch recovery scheme was proposed by Zienckiewicz and Zhu [15], in which recovery of improved stresses was obtained by interpolating from a stress surface fitted to the superconvergent stress points surrounding the node of interest. A post-processing procedure based upon the least square fitting of the displacement field over an element patch was proposed by Ahmed et al. [16]. Chen and Chen [17] proposed a modified patch recovery method to recover linear finite element solutions and showed superconvergence in the $L_{2}$ norm in a Delaunay Tessellations mesh for an arbitrary three-dimensional bounded 
domain. Zhang et al. [18] used a patch-based approximation technique to convert the interelement discontinuous field into a continuous field and implemented the adaptive remeshing procedure to distribute the error uniformly over the domain. A simple error indicator for the element free Galerkin (EFG) method and its effective performance on elasticity problems was presented by Gavete et al. [19]. Kim and $\mathrm{Li}$ [20] proposed an efficient error estimator considering non-conforming triangular and quadrilateral finite elements for elasticity problems. Nadal et al. [21] proposed an explicit-type recovery error estimator in the energy norm for the linear elasticity problem using smooth solution. Abbas and Abdul Rahman [22] proposed an energy norm based error estimator for a dual hp-adaptive procedure used in the numerical assessment of fluid-structure interaction. Ulku et al. [23] developed a recoverytype a posteriori error estimator for gradient elasticity by revisiting basic equations of the Aifantis gradients elasticity theory. An extended finite element simulation of two-dimensional (2-D) cracks and material interfaces was presented by $\mathrm{Yu}$ and Bui [24] by combining an a posteriori error estimation algorithm, a local non-conformal mesh connection strategy, and local enrichment. An hp-adaptive finite element analysis based on the discontinuous Galerkin error estimator was carried out by Bird et al. [25]. The performance of the hp-adaptive scheme was compared with a uniform and h-refinement adaptive scheme achieving the exponential rates of convergence. Various authors proposed procedures for the recovery of post-processed displacement field or their derivatives. Mirzaei [26] provided a comprehensive analysis of error estimation considering the moving least square approximation. An adaptive finite element/meshless coupling procedure based on local maximum entropy shape functions was proposed by Ullah et al. [27] for linear and non-linear problems. They used the Zienkiewicz and Zhu error estimation in the FE region and the Chung and Belytschko error estimation procedure was used in the EFG region. Parret-Fréaud et al. [28] presented a moving least squares (MLS) recovery-based procedure to obtain a smoothed stress field in which the continuity of the smoothed field is provided by shape functions of the underlying mesh.

Meshless methods, in which interpolations are free of element mesh requirements, are being developed due to the imitation of the finite element method (FEM) for situations in which distortion of elements occur, such as for large domain changes and domain discontinuities. Numerical errors are introduced in the meshless methods principally because integration cells do not match the shape function supports, while in the FEM the errors are introduced basically due to domain discretization [29]. The errors in the solution, quantified in an appropriate norm, may be controlled by using the nodal adaption in meshless methods or element adaption in the FEM employing an error estimator. The most popular type of error estimator for the finite element method is recovery or post-processing type of error estimator. The recovery procedures are based on the least square fitting of state variable or derivative of state variable by a higher order polynomial over element or node patches of mesh dependent or mesh independent local domains. Chung and Belytschko [30] measured local and global errors in the EFG method. The essence of error quantification is to use the difference between the recovered values of the stress and directly obtained EFG stress solution. The recovery of stress is obtained with reduced supports on the same nodal point of the original. Lee and Zhou [31] compared the effectiveness of different recovery schemes developed for EFG methods. The recovery schemes they compared are the discrete MLS-stationary least square fitting scheme, the continuous MLS-stationary least square scheme and the MLS-moving least square scheme. The authors concluded that the discrete MLS-stationary least square scheme outperformed the other recovery schemes. A node based error estimator for the EFG method was presented by $\mathrm{He}$ et al. [32] to reduce the spurious oscillation in regions with large stress gradients. The mesh independent recovery technique for the FEM is of relatively recent interest. Ahmed et al. [33] developed EFG based recovery techniques for finite element analysis employing mesh independent node patches. 
The development of mesh independent recovery methods with a flexibility of patch size motivated the author to use the mesh independent approach to the finite element solution of the recovery of the displacement and to present a comparison of the mesh independent recovery of displacement with that of mesh dependent recovery of the displacement. The existing recovery techniques in the FEM mostly deal with the recovery of stress over mesh dependent node patches. The present study aimed to use a recovery of the displacement field utilizing the moving least squares (MLS) procedure, which is robust and provides completeness and continuity [34] over a patch of mesh independent nodes (EFG approach) as well as the recovery of the displacement field employing the least square approach over a mesh dependent element patches for formulating an a posteriori error estimator. The global and local errors of the finite element solution are computed directly from the post-processed displacement in terms of the energy and the $\mathrm{L}_{2}$ norm. The performance of the displacement extraction based error estimator is tested on benchmark elastic plate problems. The linear and quadratic triangular elements are used for the problem domain discretization. Recovered displacement error in the $\mathrm{L}_{2}$ norm using the least square and MLS approach in addition to the energy norm for assessing the performance of the error estimation is proposed in this study. The performance of the mesh independent displacement recovery based error estimation, in both the energy and the $\mathrm{L}_{2}$ norm, is compared with that of the mesh dependent displacement recovery based error estimation in terms of effectivity, rate of convergence and adaptively refined meshes.

\section{Problem formulation}

Two-dimensional linear elastic problems with stress field $\sigma$ and unknown displacement field $\boldsymbol{u}$, in a domain $\Omega$ bounded by $\Gamma=\Gamma_{\mathrm{t}} \cup \Gamma_{\mathrm{u}}$, are found by satisfying the following equilibrium equation:

$$
\boldsymbol{L}^{T} \boldsymbol{\sigma}+\boldsymbol{f}=0 \text { in } \Omega,
$$

and boundary conditions:

$$
\begin{aligned}
& \boldsymbol{\sigma} \cdot \boldsymbol{n}=\bar{t} \text { on } \Gamma_{\mathrm{t}}, \\
& \boldsymbol{u}=\bar{u} \quad \text { on } \Gamma_{\mathrm{u}},
\end{aligned}
$$

where $\boldsymbol{f}$ is the force vector, $\boldsymbol{L}^{\boldsymbol{T}}$ is the derivative operator, $\bar{t}$ and $\bar{u}$ are prescribed tractions and displacements on $\Gamma_{\mathrm{t}}$ and $\Gamma_{\mathrm{u}}$, respectively, and $\boldsymbol{n}$ is the unit outward normal on boundary $\Gamma=\Gamma_{\mathrm{t}}$ $\cup \Gamma_{\mathrm{u}}$.

Strain vector $(\boldsymbol{\varepsilon})$ and the constitutive relation are written as

$$
\begin{aligned}
& \boldsymbol{\varepsilon}=\boldsymbol{L} \boldsymbol{u}, \\
& \boldsymbol{\sigma}=\boldsymbol{D} \varepsilon,
\end{aligned}
$$

where $\boldsymbol{D}$ is the elasticity matrix of a linear isotropic material,

By finite element discretization, displacements $(\boldsymbol{u})$ of any point within an element are calculated based on the following equation:

$$
u=N d,
$$

where $\boldsymbol{N}$ is the matrix of the interpolation functions, also known as shape functions, and $\boldsymbol{d}$ is the nodal displacement matrix. Using Equation 6, the strains can be related to the nodal displacements by the following formula:

$$
\varepsilon=L N d=B d,
$$

where $\boldsymbol{B}$ is the strain interpolation matrix. 
Using the standard Galerkin method yields the following matrix equation:

$$
\boldsymbol{K} \boldsymbol{d}=\boldsymbol{F}
$$

$\boldsymbol{K}$ and $\mathbf{F}$ are found by using the following equations:

$$
\begin{aligned}
& k_{i j}=\int_{\Omega} B_{i}^{T} D B_{j} \mathrm{~d} \Omega \\
& F_{i j}=\int_{\Gamma_{i}} N_{j}^{T} \bar{t}_{i} \mathrm{~d} \Gamma+\int_{\Omega} N_{j}^{T} b \mathrm{~d} \Omega
\end{aligned}
$$

where $\boldsymbol{b}$ is the body force per unit volume.

\section{Error estimation}

The error in the computed state variable, i.e. displacement $(u), e_{u}{ }^{*}\left(\right.$ or $\left.e_{\sigma}{ }^{*}\right)$, is defined as the difference between the exact (or recovered) values of $u$ (or $\sigma$ ) and respective computed values, $u^{h}$ (or $\sigma^{h}$ ), i.e.

$$
e_{u}^{*}=u-u^{h}
$$

The errors can be measured in appropriate norms. The integral measure of the error in the energy norm $(E)$ and the $\mathrm{L}_{2}$ norm $\left(L_{2}\right)$ may be defined as follows:

$$
\begin{aligned}
& \|e\|_{E}=\left[\int_{\Omega} e_{\sigma}^{* T} D e_{\sigma}^{*} \mathrm{~d} \Omega\right]^{1 / 2} \text { and } \\
& \|e\|_{L_{2}}=\left[\int_{\Omega} e_{u}^{* T} e_{u}^{*} \mathrm{~d} \Omega\right]^{1 / 2}, \text { respectively. }
\end{aligned}
$$

An estimator is asymptotically exact for a particular problem if the problem global and local (element) effectivity index $(\theta)$, i.e. the ratio of estimated error to true error, converges to one when the mesh size approaches zero [11].

$$
\theta=\frac{\|e\|}{\left\|e_{e x}\right\|}
$$

where $\left\|e_{e x}\right\|$ and $\|e\|$ denote the exact error and the evaluated error estimate in the energy or the $\mathrm{L}_{2}$ norm, respectively.

Accuracy $\eta$ of a finite element solution may be defined as follows:

$$
\eta=\frac{\left\|e^{*}\right\|}{\left\|\sigma^{*}\right\|}
$$

The solution is acceptable if $\eta \leq \eta_{\text {allow }}$, where $\eta_{\text {allow }}$ is the allowable accuracy. If not, hrefinement i.e. modification of the element size is needed.

\section{Mesh independent displacement recovery}

In the mesh independent recovery i.e. the moving least square (MLS) interpolation technique, three components are used to express a function $u(x)$ with the approximation $u^{h}(x)$, a weight function $w(x)$ associated to each node, a basis function $P(x)$, usually consisting of a polynomial, and a set of coefficients $a(x)$, which are functions of the coordinates. Let the nodes be defined by $x_{1} \ldots x_{n}$, where $x_{i}=\left(x_{i}, y_{i}\right)$ in two dimensions. The MLS $u^{h}(x)$ approximation can be defined as

$$
u^{h}(x)=\sum_{i=1}^{m} \phi\left(x_{i}\right) u_{i}=\phi(x) u
$$


where $\phi(x)$ is the vector of the shape function and $m$ is the total number of terms in the basis function. The basis function $P(x)$ with $m$ as six is given as

$$
P^{T}(x)=\left[1, x, y, x^{2}, x y, y^{2}\right]
$$
follows:

The vector of coefficients $\boldsymbol{a}(x)$ can be obtained by minimizing a weighted residual as

$$
J=\sum_{i=1}^{n} w\left(x-x_{i}\right)\left[P^{T}\left(x_{i}\right) a(x)-u_{i}^{h}\right]^{2}
$$

where $n$ is the number of nodes $i$ and $w\left(x-x_{i}\right)$ is a weight function in 2D, associated to each node (domain of influence of that node, Figure 1). The weight function is usually built in such a way that it takes a unit value near the point where the function and its derivatives are to be computed and vanishes outside a region $\Omega$ i surrounding the point $x_{i}$. The following cubic spline weight function with a circular domain of influence is considered in the present study,

$$
w(x-x i)=w(\bar{d})=\left\{\begin{array}{cc}
\frac{2}{3}-4 \bar{d}^{2}+4 \bar{d}^{3} & \text { for } \bar{d} \leq \frac{1}{2} \\
\frac{4}{3}-4 \bar{d}+4 \bar{d}^{2}-\frac{4}{3} \bar{d}^{3} & \text { for } 1 \leq \bar{d} \leq \frac{1}{2} \\
0 & \text { for } \bar{d}>1
\end{array}\right\}
$$

where $\bar{d}=\|x-x i\| / d_{w}$ and $d_{w}$ is the size of the influence domain of point $x_{i}$.

Minimization of the weighted residual leads to the well-known form of the shape function equation:

$$
\phi(x)=P^{T}(x) A^{-1}(x) B(x) \phi^{h}=N^{T}(x) \phi^{h}
$$

where $N^{T}(x)$ contains the shape functions of nodes and $\phi^{h}$ is the vector of nodal parameters of the field variables.

For a certain shape function $\phi_{i}(x)$ of node $i$ at a point is given as

$$
\phi_{i}(x)=P^{T}(x) A^{-1}(x) P\left(x_{i}\right) B(x)
$$

The matrices $\mathrm{A}(\mathrm{x})$ and $\mathrm{B}(\mathrm{x})$ are given as

$$
\begin{aligned}
& A(x)=\sum_{i=0}^{n} w_{i}\left(x-x_{i}\right) P\left(x_{i}\right) P^{T}\left(x_{i}\right) \\
& B(x)=\left[w_{i}\left(x-x_{i}\right) P\left(x_{i}\right), \ldots \ldots, \ldots w_{n}\left(x-x_{n}\right) P\left(x_{n}\right)\right]
\end{aligned}
$$

The derivatives of the weight function with respect to $x_{i}$ can be found using the chain rule of differentiation.

The vector $\boldsymbol{a}(x)$ can be written as

$$
\boldsymbol{a}(x)=A^{-1}(x) B(x) \phi^{h}
$$

\section{- Field nodes}

Support domains

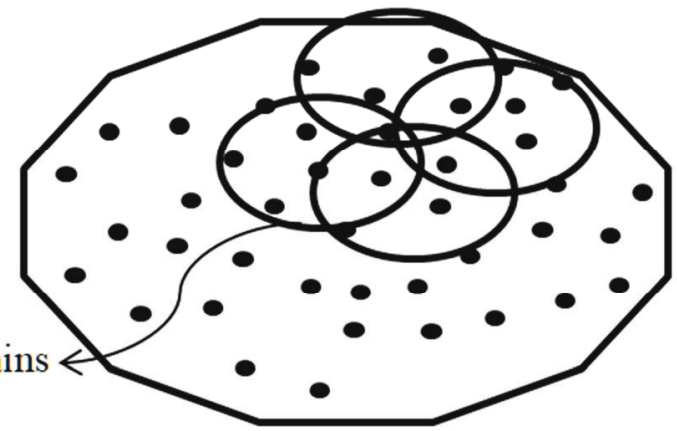

Fig. 1 Node influence (support) domains for the mesh independent (MLS) recovery technique 


\section{Mesh dependent displacement recovery}

The recovery of the displacement field is obtained by the least square (LS) fitting of the computed nodal displacement using a higher order polynomial over an element patch in a mesh around the element under consideration (Figure 2). To perform the least square fitting, the following functional is minimized:

$$
\pi_{f}(\boldsymbol{a})=\frac{1}{2} \sum_{i=1}^{n p}\left[u_{i}^{h}\left(x_{i}, y_{i}\right)-u\left(x_{i}, y_{i}\right)\right]^{2}
$$

where $\boldsymbol{u}_{\boldsymbol{i}}\left(x_{i}, y_{i}\right)=\boldsymbol{P}_{\boldsymbol{i}}\left(x_{i}, y_{i}\right) \times \boldsymbol{a}$

$$
\boldsymbol{u}_{\boldsymbol{i}}=\left[\begin{array}{ll}
u_{i} & v_{i}
\end{array}\right]^{\mathrm{T}}, \boldsymbol{a}=\left[\begin{array}{ll}
a_{u} & a_{v}
\end{array}\right]^{\mathrm{T}} .
$$

where $u_{i}$ and $v_{i}$ are nodal parameters of the field variables in the $x$ and $y$ directions and $\boldsymbol{a}$ is the vector of unknown parameters $a_{u}$ and $a_{v}$.

$$
\begin{aligned}
& \boldsymbol{P}_{i}=\left[\begin{array}{cc}
\boldsymbol{p}_{\boldsymbol{i}} & 0 \\
0 & \boldsymbol{p}_{\boldsymbol{i}}
\end{array}\right] \\
& \boldsymbol{p}_{\boldsymbol{i}}=\left[1, x_{i}, y_{i}, x_{i}^{2}, x_{i} y_{i}, y_{i}^{2}, \ldots \ldots\right]
\end{aligned}
$$

where $\left(x_{i}, y_{i}\right)$ are coordinates of the sampling points. The total number of sampling points $n p$ is equal to the total number of nodes in the patch.

The minimization condition of $\pi_{\mathrm{f}}(\boldsymbol{a})$ implies that $\boldsymbol{a}$ satisfies the following relation:

$$
\sum_{i=1}^{n p} P_{i}^{T}\left(x_{i}, y_{i}\right) \cdot P_{i}\left(x_{i}, y_{i}\right) \cdot \boldsymbol{a}=\sum_{i=1}^{n p} P_{i}^{T}\left(x_{i}, y_{i}\right) \times u_{i}^{h}\left(x_{i}, y_{i}\right)
$$

After having solved $\boldsymbol{a}$, the following relation is obtained.

$$
\begin{aligned}
& \boldsymbol{a}=\boldsymbol{A}^{-1} \boldsymbol{b} \\
& \text { where } \boldsymbol{A}=\sum_{i=1}^{n p} P_{i}^{T}\left(x_{i}, y_{i}\right) P_{i}\left(x_{i},\right. \\
& \boldsymbol{b}=\sum_{i=1}^{n p} P_{i}^{T}\left(x_{i}, y_{i}\right) u_{i}^{h}\left(x_{i}, y_{i}\right)
\end{aligned}
$$

Element under consideration
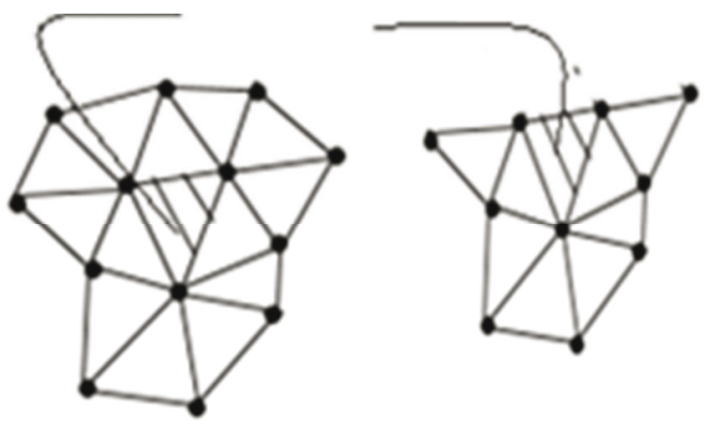

Fig. 2 Element patches for mesh dependent recovery techniques

\section{Numerical examples}

\subsection{Square plate problem}

The performance of the displacement recovery and error estimation is demonstrated through the recovery based finite element analysis of an elastic 2D membrane plate taken as an example. The example was used by Zienkiewicz and Zhu [15] to demonstrate the 
effectiveness of the $\mathrm{ZZ}$ error estimation. The benchmark example considers an infinite domain problem where a $1 \times 1$ square domain is extracted and centred at the origin of the coordinates with body loads $\left(b_{x}, b_{y}\right)$ over the domain. The corresponding Neumann boundary conditions are imposed. The known exact displacement solution $(u, v)$ and body loads in the form of $2^{\text {rd }}$ order polynomials are given in Equations 31 to 33.

$$
\begin{aligned}
& u=0 ; v=-x y(1-x)(1-y) \\
& b_{x}=(\alpha+\beta)(1-2 x)(1-2 y) \\
& b_{y}=-2 \beta y(1-y)-(\alpha+2 \beta) 2 x(1-x)
\end{aligned}
$$

The constants $\alpha$ and $\beta$ are given as

$$
\alpha=E v /[(1-2 v)(1+v)] ; \beta=E /[2(1+v)]
$$

where $E$ and $v$ are the modulus of elasticity and Poisson's ratio, respectively, with a value of $1.0 \mathrm{~N} / \mathrm{mm}^{2}$ and 0.3 .

The benchmark problem domain is discretized as uniform mesh and non-uniform mesh using linear and quadratic triangular elements. The initial structured and unstructured meshes are shown in Figure 3. The solution error convergence with order of refinement in the finite element analysis and the effectivity of error estimation using the mesh based displacement recovery and meshless displacement recovery for linear elements are given in Tables 1-4. The results of the analysis of error convergence and effectivity using quadratic elements are shown in Tables 5-8. The solution errors are presented in two alternative norms, i.e. the energy and the $\mathrm{L}_{2}$ norm. A minimum of five levels of mesh fineness are used in uniform and non-uniform meshing. The convergence rates of the solution error presented in the $\mathrm{L}_{2}$ norm with uniform refinement of linear and quadratic elements in the original solution, the post-processed solution using mesh based recovery using LS interpolation, the meshless recovery and the mesh based recovery using MLS interpolation are found to be 1.93939, 2.15385, 2.06073, 2.07087 and $3.047331,3.7214993 .83913,3.84449$, respectively. For linear and quadratic elements, the convergence rates of error in the energy norm are $0.97875,1.88603,2.28605$, 2.01082 and $1.978481,2.88449,3.19899,3.31935$, respectively.

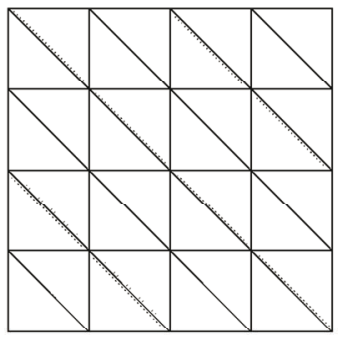

3(a): Structured mesh

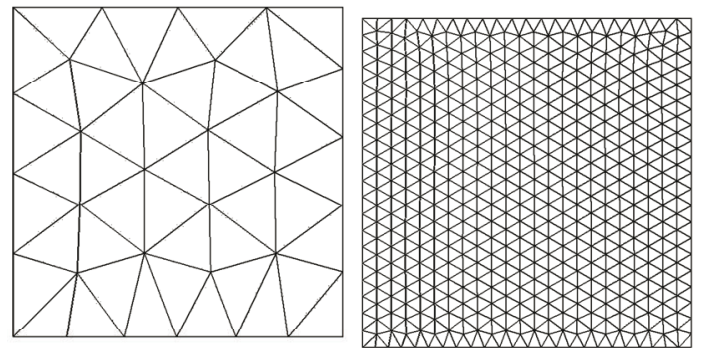

3(b): Unstructured mesh

Fig. 3 Square plate problem domain discretization 
Table 1 Error convergence and global effectivity for plate problem using displacement recovery (structured linear element mesh, $\mathrm{L}_{2}$ norm)

\begin{tabular}{|c|c|c|c|c|c|c|c|}
\hline & \multicolumn{7}{|c|}{ Error and effectivity } \\
\cline { 2 - 8 } $\begin{array}{c}\text { Mesh size } \\
(1 / \mathrm{h})\end{array}$ & $\begin{array}{c}\text { FEM } \\
\left(\mathrm{x} 10^{-3}\right)\end{array}$ & $\begin{array}{c}\text { Mesh based } \\
\text { LS } \\
\text { recovery } \\
\left(\mathrm{x} 10^{-3}\right)\end{array}$ & $\begin{array}{c}\text { Effectivity } \\
(\theta)\end{array}$ & $\begin{array}{c}\text { Mesh } \\
\text { independent } \\
\text { MLS recovery } \\
\left(\mathrm{x} 10^{-3}\right)\end{array}$ & $\begin{array}{c}\text { Effectivity } \\
(\theta)\end{array}$ & $\begin{array}{c}\text { Mesh based } \\
\text { MLS } \\
\text { recovery } \\
\left(\mathrm{x} 10^{-3}\right)\end{array}$ & $\begin{array}{c}\text { Effectivity } \\
(\theta)\end{array}$ \\
\hline $1 / 4$ & 5.36521 & 3.78408 & 0.709497 & 3.05484 & 0.793014 & 3.13677 & 0.647411 \\
\hline $1 / 8$ & 1.46955 & 0.81181 & 0.690517 & 0.68201 & 0.690152 & 0.71683 & 0.663645 \\
\hline $1 / 12$ & 0.66623 & 0.33314 & 0.682793 & 0.29994 & 0.679458 & 0.30878 & 0.668820 \\
\hline $1 / 16$ & 0.37753 & 0.18018 & 0.679482 & 0.16835 & 0.676664 & 0.17148 & 0.670996 \\
\hline $1 / 24$ & 0.16872 & 0.07739 & 0.676790 & 0.07478 & 0.675119 & 0.07547 & 0.672721 \\
\hline $1 / 32$ & 0.09509 & 0.04294 & 0.675747 & 0.04207 & 0.674692 & 0.04230 & 0.673370 \\
\hline Rate of & 1.93939 & 2.15385 & & 2.06073 & & 2.07087 & \\
conv. & & & & & & & \\
\hline
\end{tabular}

Table 2 Error convergence and global effectivity for plate problem using displacement recovery (structured linear element mesh, energy norm)

\begin{tabular}{|c|c|c|c|c|c|c|c|}
\hline \multirow{2}{*}{$\begin{array}{c}\text { Mesh } \\
\text { size } \\
(1 / \mathrm{h})\end{array}$} & $\begin{array}{c}\text { FEM } \\
\left(\mathrm{x} 10^{-3}\right)\end{array}$ & $\begin{array}{c}\text { Mesh based } \\
\text { LS } \\
\text { recovery } \\
\left(\mathrm{x} 10^{-3}\right)\end{array}$ & $\begin{array}{c}\text { Effectivity, } \\
(\theta)\end{array}$ & $\begin{array}{c}\text { Mesh } \\
\text { independent } \\
\text { MLS recovery } \\
\left(\mathrm{x} 10^{-3}\right)\end{array}$ & $\begin{array}{c}\text { Effectivity, } \\
(\theta)\end{array}$ & $\begin{array}{c}\text { Mesh based } \\
\text { MLS } \\
\text { recovery } \\
\left(\mathrm{x} 10^{-3}\right)\end{array}$ & $\begin{array}{c}\text { Effectivity, } \\
(\theta)\end{array}$ \\
\hline $1 / 4$ & 93.74913 & 58.47843 & 0.929995 & 43.86287 & 0.923637 & 44.65509 & 0.877835 \\
\hline $1 / 8$ & 48.44923 & 16.79087 & 0.968643 & 8.37827 & 0.973875 & 11.55328 & 0.956266 \\
\hline $1 / 12$ & 32.51236 & 7.77119 & 0.983109 & 3.32995 & 0.987285 & 5.06279 & 0.977898 \\
\hline $1 / 16$ & 24.44091 & 4.46214 & 0.989587 & 1.74236 & 0.992567 & 2.80995 & 0.986748 \\
\hline $1 / 24$ & 16.32114 & 2.02873 & 0.994934 & 0.70853 & 0.996584 & 1.22637 & 0.993723 \\
\hline $1 / 32$ & 12.24803 & 1.15809 & 0.997014 & 0.37808 & 0.998051 & 0.68221 & 0.996356 \\
\hline Rate of & 0.97875 & 1.88603 & & 2.28605 & & 2.01082 & \\
conv. & 0.97862 & & & & & \\
\hline
\end{tabular}

Table 3 Error convergence and global effectivity $(\theta)$ for plate problem using displacement recovery (unstructured linear element mesh, $\mathrm{L}_{2}$ norm)

\begin{tabular}{|c|c|c|c|c|c|c|c|c|}
\hline \multicolumn{2}{|c|}{ Mesh } & \multicolumn{7}{|c|}{ Error in $\mathrm{L}_{2}$ norm } \\
\hline $\begin{array}{c}\text { No. of } \\
\text { elem. }\end{array}$ & DOF & $\begin{array}{c}\text { FEM } \\
\left(\mathrm{x} 10^{-3}\right)\end{array}$ & $\begin{array}{c}\text { Mesh based } \\
\text { LS } \\
\text { recovery } \\
\left(\mathrm{x} 10^{-3}\right)\end{array}$ & $\theta$ & $\begin{array}{c}\text { Mesh } \\
\text { independent } \\
\text { MLS recovery } \\
\left(\mathrm{x} 10^{-3}\right)\end{array}$ & $\theta$ & $\begin{array}{c}\text { Mesh based } \\
\text { MLS } \\
\text { recovery } \\
\left(\mathrm{x} 10^{-3}\right)\end{array}$ & $\theta$ \\
\hline 45 & 66 & 2.71869 & 1.85310 & 1.039810 & 1.20814 & 1.147404 & 1.39813 & 0.96551 \\
\hline 88 & 118 & 1.47472 & 0.81667 & 0.993203 & 0.67965 & 1.185746 & 0.66541 & 0.95090 \\
\hline 223 & 270 & 0.55133 & 0.23674 & 0.954800 & 0.23262 & 1,154244 & 0.20895 & 0.93456 \\
\hline 925 & 1014 & 0.10963 & 0.04527 & 1.064255 & 0.04052 & 1.123506 & 0.04384 & 1.06037 \\
\hline 1978 & 2106 & 0.04908 & 0.01943 & 1.095941 & 0.01758 & 1.123434 & 0.01900 & 1.09405 \\
\hline
\end{tabular}


Table 4 Error convergence and global effectivity $(\theta)$ for plate problem using displacement recovery (unstructured linear element mesh, energy norm)

\begin{tabular}{|c|c|c|c|c|c|c|c|c|}
\hline \multicolumn{2}{|c|}{ Mesh } & \multicolumn{7}{|c|}{ Error in energy norm } \\
\hline $\begin{array}{c}\text { No. of } \\
\text { elem. }\end{array}$ & DOF & $\begin{array}{c}\text { FEM } \\
\left(\mathrm{x} 10^{-3}\right)\end{array}$ & $\begin{array}{c}\text { Mesh based } \\
\text { LS } \\
\text { recovery } \\
\left(\mathrm{x} 10^{-3}\right)\end{array}$ & $\theta$ & $\begin{array}{c}\text { Mesh } \\
\text { independent } \\
\text { MLS recovery } \\
\left(\mathrm{x} 10^{-3}\right)\end{array}$ & $\theta$ & $\begin{array}{c}\text { Mesh based } \\
\text { MLS } \\
\text { recovery } \\
\left(\mathrm{x} 10^{-3}\right)\end{array}$ & $\theta$ \\
\hline 45 & 66 & 67.89001 & 44.07944 & 0.99671 & 25.32200 & 0.97832 & 33.50533 & 0.93269 \\
\hline 88 & 118 & 50.17756 & 21.90273 & 0.97577 & 15.70293 & 0.98731 & 17.19762 & 0.95312 \\
\hline 223 & 270 & 30.64371 & 8.43683 & 0.97830 & 6.53116 & 0.98927 & 6.80270 & 0.97306 \\
\hline 925 & 1014 & 13.87145 & 1.89632 & 0.99341 & 1.23394 & 0.99402 & 1.66148 & 0.99301 \\
\hline 1978 & 2106 & 9.28878 & 0.89337 & 0.99580 & 0.52999 & 0.99643 & 0.77846 & 0.99565 \\
\hline
\end{tabular}

Table 5 Error convergence and global effectivity for plate problem using displacement recovery (structured quadratic element mesh, $\mathrm{L}_{2}$ norm)

\begin{tabular}{|c|c|c|c|c|c|c|c|}
\hline \multirow{2}{*}{$\begin{array}{c}\text { Mesh size } \\
(1 / \mathrm{h})\end{array}$} & $\begin{array}{c}\text { FEM } \\
\left(\mathrm{x} 10^{-3}\right)\end{array}$ & $\begin{array}{c}\text { Mesh based } \\
\text { LS } \\
\text { recovery } \\
\left(\mathrm{x} 10^{-3}\right)\end{array}$ & $\begin{array}{c}\text { Effectivity } \\
(\theta)\end{array}$ & $\begin{array}{c}\text { Mesh } \\
\text { independent } \\
\text { MLS recovery } \\
\left(\mathrm{x} 10^{-3}\right)\end{array}$ & $\begin{array}{c}\text { Effectivity } \\
(\theta)\end{array}$ & $\begin{array}{c}\text { Mesh based } \\
\text { MLS } \\
\text { recovery } \\
\left(\mathrm{x} 10^{-3}\right)\end{array}$ & $\begin{array}{c}\text { Effectivity } \\
(\theta)\end{array}$ \\
\hline $1 / 4$ & 0.24489 & 1.03015 & 4.24214 & 0.03358 & 0.95715 & 0.04318 & 0.93427 \\
\hline $1 / 8$ & 0.02887 & 0.08912 & 3.17330 & 0.00259 & 0.98523 & 0.00326 & 0.97639 \\
\hline $1 / 12$ & 0.00842 & 0.01930 & 2.44485 & 0.00054 & 0.99301 & 0.00068 & 0.98850 \\
\hline $1 / 16$ & 0.00353 & 0.00638 & 2.02200 & 0.00017 & 0.99599 & 0.00022 & 0.99328 \\
\hline $1 / 24$ & 0.00104 & 0.00132 & 1.58496 & 0.00004 & 0.99821 & 0.00004 & 0.99692 \\
\hline $\begin{array}{c}\text { Rate of } \\
\text { conv. }\end{array}$ & 3.04733 & 3.721499 & & 3.83913 & & 3.84449 & \\
\hline
\end{tabular}

Table 6 Error convergence and global effectivity $(\theta)$ for plate problem using displacement recovery (structured quadratic element mesh, energy norm)

\begin{tabular}{|c|c|c|c|c|c|c|c|}
\hline \multirow{2}{*}{$\begin{array}{c}\text { Mesh size } \\
(1 / \mathrm{h})\end{array}$} & $\begin{array}{c}\text { FEM } \\
\left(\mathrm{x} 10^{-3}\right)\end{array}$ & $\begin{array}{c}\text { Mesh based } \\
\text { LS } \\
\text { recovery } \\
\left(\mathrm{x} 10^{-3}\right)\end{array}$ & $\theta$ & $\begin{array}{c}\text { Mesh } \\
\text { independent } \\
\text { MLS recovery } \\
\left(\mathrm{x} 10^{-3}\right)\end{array}$ & $\theta$ & $\begin{array}{c}\text { Mesh based } \\
\text { MLS recovery } \\
\left(\mathrm{x} 10^{-3}\right)\end{array}$ & $\theta$ \\
\hline $1 / 4$ & 13.16827 & 12.25770 & 1.25556 & 0.30250 & 0.99899 & 0.53221 & 1.00035 \\
\hline $1 / 8$ & 3.38825 & 1.72016 & 1.08271 & 0.03751 & 1.00000 & 0.05530 & 1.00067 \\
\hline $1 / 12$ & 1.51492 & 0.52885 & 1.04045 & 0.01003 & 1.00007 & 0.01431 & 1.00042 \\
\hline $1 / 16$ & 0.85400 & 0.22787 & 1.02434 & 0.00384 & 1.00006 & 0.00545 & 1.00027 \\
\hline $1 / 24$ & 0.38016 & 0.06980 & 1.01265 & 0.00098 & 1.00004 & 0.00139 & 1.00014 \\
\hline $\begin{array}{c}\text { Rate of } \\
\text { conv. }\end{array}$ & 1.97848 & 2.88449 & & 3.19899 & & 3.31935 & \\
\hline
\end{tabular}


Table 7 Error convergence and global effectivity $(\theta)$ for plate problem using displacement recovery (unstructured quadratic element mesh, $\mathrm{L}_{2}$ norm)

\begin{tabular}{|c|c|c|c|c|c|c|c|c|}
\hline \multicolumn{2}{|c|}{ Mesh } & \multicolumn{7}{|c|}{ Error in $\mathrm{L}_{2}$ norm } \\
\hline $\begin{array}{c}\text { No. of } \\
\text { elem. }\end{array}$ & DOF & $\begin{array}{c}\text { FEM } \\
\left(\mathrm{x} 10^{-3}\right)\end{array}$ & $\begin{array}{c}\text { Mesh based } \\
\text { LS } \\
\text { recovery } \\
\left(\mathrm{x} 10^{-3}\right)\end{array}$ & $\theta$ & $\begin{array}{c}\text { Mesh } \\
\text { independent } \\
\text { MLS recovery } \\
\left(\mathrm{x} 10^{-3}\right)\end{array}$ & $\theta$ & $\begin{array}{c}\text { Mesh based } \\
\text { MLS } \\
\text { recovery } \\
\left(\mathrm{x} 10^{-3}\right)\end{array}$ & $\theta$ \\
\hline 45 & 220 & 0.12936 & 0.41055 & 3.26779 & 0.01755 & 0.98343 & 0.01953 & 0.98842 \\
\hline 88 & 410 & 0.04435 & 0.15772 & 3.62961 & 0.00606 & 0.98580 & 0.00593 & 0.98721 \\
\hline 223 & 984 & 0.10565 & 0.02186 & 2.19821 & 0.00104 & 0.99232 & 0.00102 & 0.99524 \\
\hline 925 & 3876 & 0.00122 & 0.00139 & 1.47747 & 0.00012 & 0.99600 & 0.00011 & 0.99888 \\
\hline 1978 & 8166 & 0.00385 & 0.00085 & 1.42296 & 0.00004 & 0.99740 & 0.00003 & 1.00008 \\
\hline
\end{tabular}

Table 8 Error convergence and global effectivity $(\theta)$ for plate problem using displacement recovery (unstructured quadratic element mesh, energy norm)

\begin{tabular}{|c|c|c|c|c|c|c|c|c|}
\hline \multicolumn{2}{|c|}{ Mesh } & \multicolumn{7}{|c|}{ Error in energy norm } \\
\hline $\begin{array}{c}\text { No. of } \\
\text { elem. }\end{array}$ & DOF & $\begin{array}{c}\text { FEM } \\
\left(\times 10^{-3}\right)\end{array}$ & $\begin{array}{c}\text { Mesh based } \\
\text { LS } \\
\text { recovery } \\
\left(\times 10^{-3}\right)\end{array}$ & $\theta$ & $\begin{array}{c}\text { Mesh } \\
\text { independent } \\
\text { MLS recovery } \\
\left(\mathrm{x} 10^{-3}\right)\end{array}$ & $\theta$ & $\begin{array}{c}\text { Mesh based } \\
\text { MLS } \\
\text { recovery } \\
\left(\times 10^{-3}\right)\end{array}$ & $\theta$ \\
\hline 45 & 220 & 6.95319 & 7.94865 & 1.42861 & 0.28504 & 0.99739 & 0.33211 & 0.99859 \\
\hline 88 & 410 & 3.45292 & 2.84782 & 1.24041 & 0.14183 & 0.99890 & 0.12038 & 0.99943 \\
\hline 223 & 984 & 1.32823 & 0.67455 & 1.09216 & 0.04689 & 0.99873 & 0.04112 & 0.99886 \\
\hline 925 & 3876 & 0.31903 & 0.07747 & 1.02221 & 0.00995 & 0.99959 & 0.00731 & 0.99984 \\
\hline 1978 & 8166 & 0.14915 & 0.02533 & 1.01201 & 0.00584 & 1.00036 & 0.00433 & 1.00055 \\
\hline
\end{tabular}

\subsection{Infinite plate with a hole problem}

A stress concentration problem in an infinite membrane, i.e., an infinite plate with an opening of a unit radius (a) at its centre is also taken to evaluate the effectiveness of the displacement recovery procedures. The plate is analysed under the action of a unit in-plane traction applied in the x-direction. Due to symmetry, only the upper left square quadrant of the plate is modelled and discretized with linear and quadratic triangular meshing schemes (Figure 4). Along the symmetry line, shear stress and the normal displacement component are zero. Equations 34 to 36 show the analytical solution for stresses and are assumed as exact solutions for the problem presented by Zienkiewicz and Zhu [15].

$$
\begin{aligned}
& \sigma_{x}=\sigma_{\infty}\left[1-\frac{a^{2}}{r^{2}}(1.5 \cos 2 \theta-\cos 4 \theta)-1.5 \frac{a^{4}}{r^{4}} \cos 4 \theta\right] \\
& \sigma_{y}=\sigma_{\infty}\left[0-\frac{a^{2}}{r^{2}}(0.5 \cos 2 \theta-\cos 4 \theta)-1.5 \frac{a^{4}}{r^{4}} \cos 4 \theta\right] \\
& \sigma_{x y}=\sigma_{\infty}\left[0-\frac{a^{2}}{r^{2}}(0.5 \sin 2 \theta-\sin 4 \theta)-1.5 \frac{a^{4}}{r^{4}} \sin 4 \theta\right]
\end{aligned}
$$

where $r^{2}=y^{2}+x^{2}$ and $\sigma_{\infty}$ is the uniaxial traction applied at infinity. 

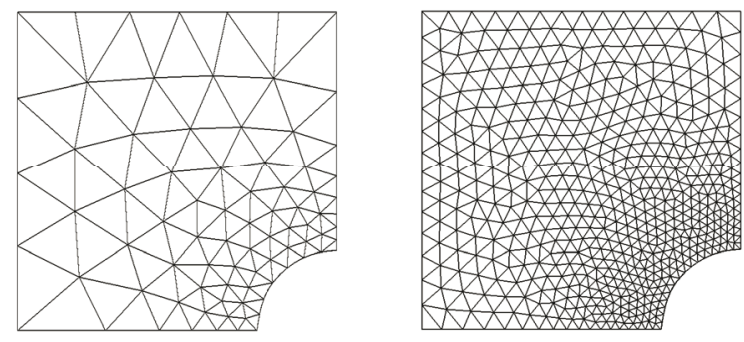

Fig. 4 Plate with hole problem domain discretization

The quality of error estimation in the stress concentration problem obtained with the techniques for mesh independent based MLS displacement recovery is compared with the techniques for mesh dependent based MLS and least square displacement recovery in terms of error convergence and global effectivity. As the problem is a typical example of stress concentration, the finite element solution error is quantified in the energy norm. The error convergence and the global effectivity of error estimation with a linear and quadratic triangular mesh using least square and MLS displacement recovery techniques are presented in Tables 9 and 10.

Table 9 Error convergence and global effectivity $(\theta)$ for plate problem with hole using displacement recovery (linear element mesh, energy norm)

\begin{tabular}{|c|c|c|c|c|c|c|c|c|}
\hline \multicolumn{2}{|c|}{ Mesh } & \multicolumn{7}{|c|}{ Error in energy norm } \\
\hline $\begin{array}{c}\text { No. of } \\
\text { elem. }\end{array}$ & DOF & $\begin{array}{c}\text { FEM } \\
\left(\mathrm{x} 10^{-3}\right)\end{array}$ & $\begin{array}{c}\text { Mesh based } \\
\text { LS } \\
\text { recovery } \\
\left(\mathrm{x} 10^{-3}\right)\end{array}$ & $\theta$ & $\begin{array}{c}\text { Mesh } \\
\text { independent } \\
\text { MLS recovery } \\
\left(\mathrm{x} 10^{-3}\right)\end{array}$ & $\theta$ & $\begin{array}{c}\text { Mesh based } \\
\text { MLS } \\
\text { recovery } \\
\left(\mathrm{x} 10^{-3}\right)\end{array}$ & $\theta$ \\
\hline 234 & 274 & 7.575 & 4.649 & 0.956607 & 3.741 & 0.908076 & 3.717 & 0.921135 \\
\hline 439 & 496 & 5.787 & 2.882 & 0.948194 & 2.461 & 0.917531 & 2.529 & 0.927395 \\
\hline 714 & 784 & 4.316 & 1.837 & 0.937876 & 1.665 & 0.933370 & 1.711 & 0.934449 \\
\hline 1002 & 1084 & 4.048 & 1.742 & 0.926739 & 1.656 & 0.921302 & 1.638 & 0.919573 \\
\hline 2415 & 2540 & 2.579 & 1.394 & 0.850214 & 1.382 & 0.845487 & 1.385 & 0.843176 \\
\hline
\end{tabular}

Table 10 Error convergence and global effectivity $(\theta)$ for plate problem with hole using displacement recovery (quadratic element mesh, energy norm)

\begin{tabular}{|c|c|c|c|c|c|c|c|c|}
\hline \multicolumn{2}{|c|}{ Mesh } & \multicolumn{7}{|c|}{ Error in energy norm } \\
\hline $\begin{array}{c}\text { No. of } \\
\text { elem. }\end{array}$ & DOF & $\begin{array}{c}\text { FEM } \\
\left(\mathrm{x} 10^{-3}\right)\end{array}$ & $\begin{array}{c}\text { Mesh based } \\
\text { LS } \\
\text { recovery } \\
\left(\mathrm{x} 10^{-3}\right)\end{array}$ & $\theta$ & $\begin{array}{c}\text { Mesh } \\
\text { independent } \\
\text { MLS recovery } \\
\left(\mathrm{x} 10^{-3}\right)\end{array}$ & $\theta$ & $\begin{array}{c}\text { Mesh based } \\
\text { MLS } \\
\text { recovery } \\
\left(\mathrm{x} 10^{-3}\right)\end{array}$ & $\theta$ \\
\hline 131 & 588 & 1.698 & 4.559 & 2.56915 & 1.469 & 0.829579 & 2.183 & 1.21923 \\
\hline 234 & 1014 & 1.510 & 3.509 & 2.18115 & 1.342 & 0.729339 & 1.311 & 0.692823 \\
\hline 439 & 1868 & 1.429 & 2.199 & 1.35794 & 1.323 & 0.488721 & 1.452 & 0.615069 \\
\hline 567 & 2392 & 1.414 & 1.695 & 0.839770 & 1.321 & 0.501294 & 1.440 & 0.619218 \\
\hline 1002 & 4170 & 1.428 & 1.495 & 0.695226 & 1.368 & 0.296206 & 1.400 & 0.340926 \\
\hline
\end{tabular}

\section{Discussion}

The benchmark problems, earlier studied by Zienckiewicz and Zhu [15] as validation problems of the $\mathrm{ZZ}$ error estimation are used to test the effectivity of the displacement recovery scheme based on error estimations. The error estimation effectivity at global and elemental levels has been compared with the errors in both the energy and the $\mathrm{L}_{2}$ norm. The 
solution errors in the energy and $\mathrm{L}_{2}$ norms presented in the tables show that the order of error obtained by employing recovery procedures is much lower than the FEM solution error, and the error convergence is also higher when compared to the FEM solution error with a reduction in the mesh size. The effect on the error order is further enhanced when higher order elements are taken for domain discretization. The effect on error convergence is reduced with higher order elements, though the rate of error convergence is still higher than the FEM error convergence with a reduction in the mesh size. While comparing the performance of the energy and $\mathrm{L}_{2}$ norms, it is found that the $\mathrm{L}_{2}$ norm used for error representation should be used with higher order element discretization for optimal performance as the effectivity, calculated in the $\mathrm{L}_{2}$ norm, converged to nearly one with higher order element discretization. The error in the energy norm is versatile and equally suited to all type of elements with various error recovery techniques. It is clear from the tables that the performance of the technique for moving least square (MLS) interpolation based displacement recovery is better than that of the technique for least square (LS) interpolation based displacement recovery employing different norm and mesh discretization. The performance of techniques for the mesh independent and mesh dependent based MLS recovery under different norms and mesh discretization is the same as the obtained values of effectivity, and the rate of convergence is the same. However, the order of error in the mesh independent MLS interpolation technique is smaller in comparison to the mesh dependent MLS interpolation technique.

The application of the displacement recovery based error estimation in guiding the error controlling strategies of the adaptive analysis is also demonstrated. Results of the adaptive refinement are obtained for the meshless displacement recovery using the MLS interpolation, the mesh dependent displacement recovery using the MLS interpolation and the mesh dependent least square (LS) interpolation procedure. Tables 11 and 12 show global errors (FEM and projected), the number of element and DOF in refined meshes, obtained from the linear and quadratic elements of the initial mesh, to bring accuracy to the target accuracy level. The global error of higher order elements, in both the energy and the $\mathrm{L}_{2}$ norm, is much lower than the global error of lower order elements. The target error of $4 \%$ is selected for adaptive finite element analyses employing linear element discretization and a target error of $1 \%$ is fixed for adaptive analyses employing quadratic elements based on the recovered global error in the problem. The computed global error for linear elements is the highest for the least square interpolation based recovery and it is minimum for the MLS interpolation based recovery technique and the error represented in the energy norm, while it is maximum for the mesh independent recovery technique and minimum for the least square interpolation based recovery technique when the error is represented in the $\mathrm{L}_{2}$ norm. For quadratic elements, the global error is the same for different recovery techniques and under different norms. The adaptively refined meshes with a target error using the least square and MLS interpolation based error estimation are given in Figures 5-12. The initial meshes are adaptively refined to bring the solution error within the target limits and to distribute the error equally among the elements. The number of elements required to achieve the target error represented in the energy norm depend on the global error. It can be concluded that the adaptive analysis based on interpolation recovery techniques may predict high errors zones and control the solution error effectively. 
Table 11 Global errors (FEM and projected) and number of element (N) with DOF of adaptively refined meshes (linear elements) using displacement recovery based analysis ( $4 \%$ target error for energy and $\mathrm{L}_{2}$ norm)

\begin{tabular}{|c|c|c|c|c|c|c|c|c|c|c|}
\hline \multirow{3}{*}{$\begin{array}{l}\text { Displace-ment } \\
\text { recovery type }\end{array}$} & \multicolumn{2}{|c|}{ Initial mesh } & \multirow{2}{*}{\multicolumn{4}{|c|}{ Energy norm }} & \multirow{2}{*}{\multicolumn{4}{|c|}{$\mathrm{L}_{2}$ norm }} \\
\hline & \multirow{2}{*}{$\begin{array}{l}\text { No. } \\
\text { of } \\
\text { elem. } \\
(\mathrm{N})\end{array}$} & \multirow{2}{*}{ DoF } & & & & & & & & \\
\hline & & & $\begin{array}{l}\text { Error } \\
\text { FEM } \\
\end{array}$ & $\begin{array}{l}\text { Error } \\
\text { proj. }\end{array}$ & $\mathrm{N}$ & DoF & $\begin{array}{l}\text { Error } \\
\text { FEM } \\
\end{array}$ & $\begin{array}{l}\text { Error } \\
\text { proj. }\end{array}$ & $\mathrm{N}$ & DoF \\
\hline \multirow{2}{*}{$\begin{array}{c}\text { Mesh } \\
\text { independent } \\
\text { recovery } \\
\text { ( MLS ) }\end{array}$} & 45 & 66 & 28.3 & 28.4 & 2609 & 2770 & 8.16 & 9.33 & 283 & 332 \\
\hline & 88 & 118 & 20.9 & 21.0 & 2890 & 3066 & 4.42 & 5.23 & 116 & 148 \\
\hline \multirow{2}{*}{$\begin{array}{l}\text { Mesh based } \\
\text { recovery } \\
(\text { MLS ) }\end{array}$} & 45 & 66 & 28.3 & 28.9 & 2468 & 2622 & 8.16 & 7.95 & 158 & 190 \\
\hline & 88 & 118 & 20.9 & 20.8 & 2731 & 2896 & 4.42 & 4.22 & 97 & 126 \\
\hline \multirow{2}{*}{$\begin{array}{l}\text { Mesh based } \\
\text { recovery } \\
(\text { LS ) }\end{array}$} & 45 & 66 & 28.8 & 32.1 & 4060 & 4254 & 8.16 & 8.60 & 141 & 617 \\
\hline & 88 & 118 & 20.9 & 21.7 & 3249 & 3432 & 4.42 & 4.41 & 87 & 114 \\
\hline
\end{tabular}

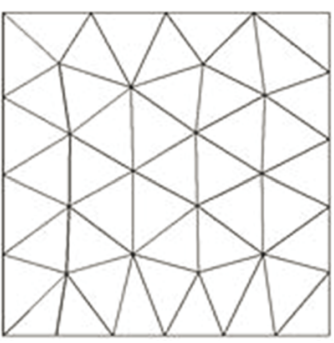

(a) Initial mesh

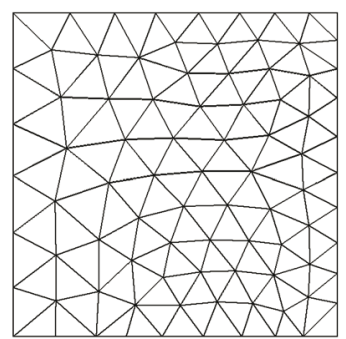

(b) Mesh based (LS)

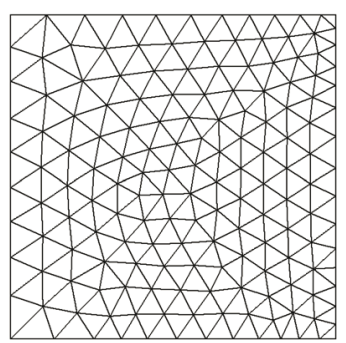

(c) Mesh independent (MLS)

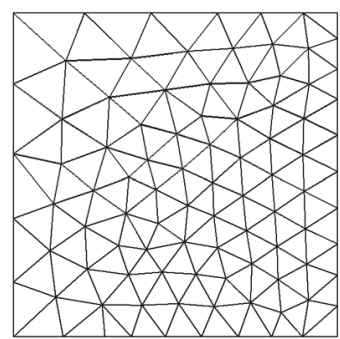

(d) Mesh based (MLS)

Fig. 5 Adaptively refined mesh in plate problem domain using displacement recovery techniques [linear initial mesh elements $=45,4 \%$ target error, $\mathrm{L}_{2}$ norm $]$

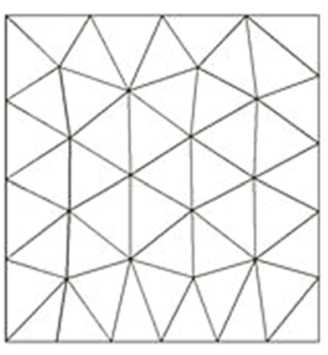

(a) Initial mesh

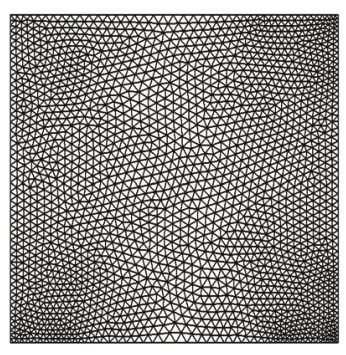

(b) Mesh based (LS)

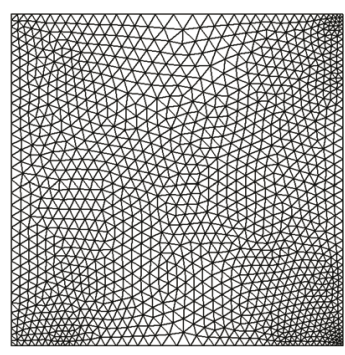

(c) Mesh independent (MLS)

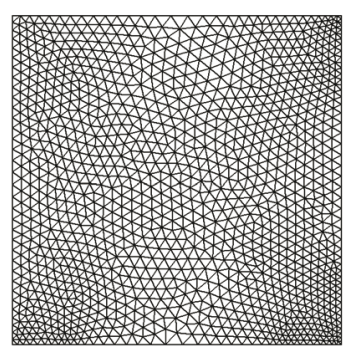

(d) Mesh based (MLS)

Fig. 6 Adaptively refined mesh in plate problem domain using displacement recovery techniques [linear initial mesh elements $=45,4 \%$ target error in energy norm]

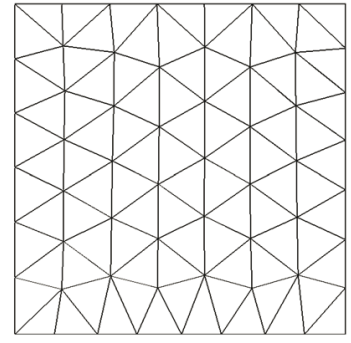

(a) Initial mesh

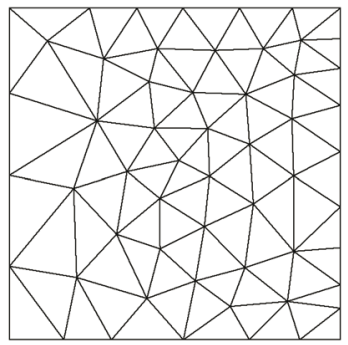

(b) Mesh based (LS)

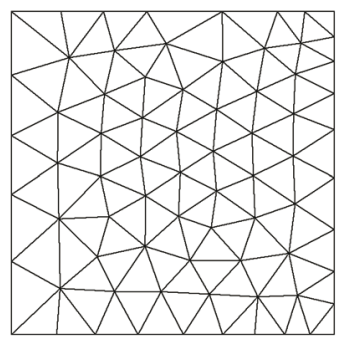

(c) Mesh independent (MLS)

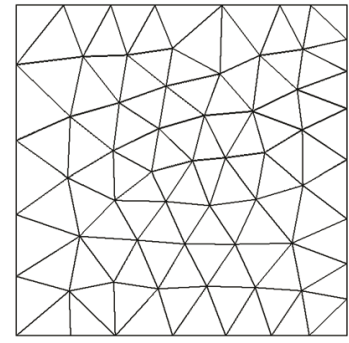

(d) Mesh based (MLS)

Fig. 7 Adaptively refined mesh in plate problem domain using displacement recovery techniques [linear initial mesh elements $=88,4 \%$ target error, $\mathrm{L}_{2}$ norm $]$ 


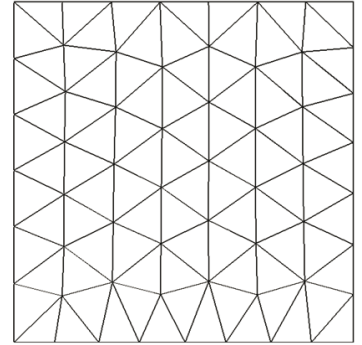

(a) Initial mesh

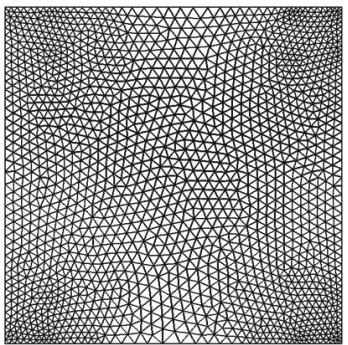

(b) Mesh based (LS)

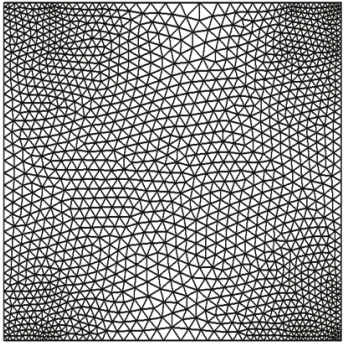

(c) Mesh independent (MLS)

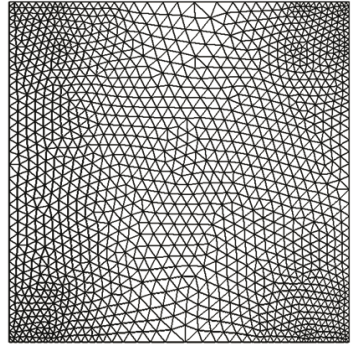

(d) Mesh based (MLS)

Fig. 8 Adaptively refined mesh in plate problem domain using displacement recovery techniques [linear initial mesh elements $=88,4 \%$ target error, energy norm]

Table 12 Global errors and number of element (n) with DOF of adaptively refined meshes (quadratic elements) using displacement recovery based analysis ( $1 \%$ target error for energy norm and $0.1 \%$ target error for $\mathrm{L}_{2}$ norm)

\begin{tabular}{|c|c|c|c|c|c|c|c|c|c|c|}
\hline \multirow{3}{*}{$\begin{array}{l}\text { Displace-ment } \\
\text { recovery type }\end{array}$} & \multicolumn{2}{|c|}{ Initial mesh } & \multirow{2}{*}{\multicolumn{4}{|c|}{ Energy norm }} & \multirow{2}{*}{\multicolumn{4}{|c|}{$\mathrm{L}_{2}$ norm }} \\
\hline & \multirow{2}{*}{$\begin{array}{l}\text { No. } \\
\text { of } \\
\text { elem. } \\
(\mathrm{N})\end{array}$} & \multirow[b]{2}{*}{ DoF } & & & & & & & & \\
\hline & & & $\begin{array}{l}\text { Error } \\
\text { FEM }\end{array}$ & $\begin{array}{l}\text { Error } \\
\text { proj. }\end{array}$ & $\mathrm{N}$ & DoF & $\begin{array}{l}\text { Error } \\
\text { FEM }\end{array}$ & $\begin{array}{l}\text { Error } \\
\text { proj. }\end{array}$ & $\mathrm{N}$ & DoF \\
\hline \multirow{2}{*}{$\begin{array}{c}\text { Mesh } \\
\text { independent } \\
\text { recovery } \\
\text { ( MLS ) }\end{array}$} & 45 & 220 & 2.9 & 2.9 & 384 & 1690 & 0.39 & 0.38 & 769 & 3284 \\
\hline & 88 & 410 & 1.5 & 1.5 & 359 & 1580 & 0.13 & 0.13 & 218 & 986 \\
\hline \multirow{2}{*}{$\begin{array}{c}\text { Mesh based } \\
\text { recovery } \\
(\text { MLS ) }\end{array}$} & 45 & 220 & 2.9 & 2.9 & 400 & 1750 & 0.39 & 0.38 & 575 & 2489 \\
\hline & 88 & 410 & 1.5 & 1.5 & 323 & 1428 & 0.13 & 0.13 & 179 & 824 \\
\hline \multirow{2}{*}{$\begin{array}{l}\text { Mesh based } \\
\text { recovery } \\
(\mathrm{LS})\end{array}$} & 45 & 220 & 2.9 & 4.3 & 1110 & 4634 & 0.39 & 1.28 & 6929 & 28197 \\
\hline & 88 & 410 & 1.4 & 1.8 & 595 & 2532 & 0.13 & 0.48 & 1398 & 5376 \\
\hline
\end{tabular}

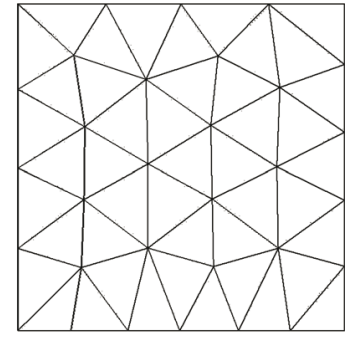

(a) Initial mesh

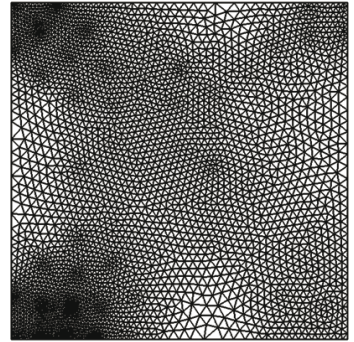

(b) Mesh based (LS)

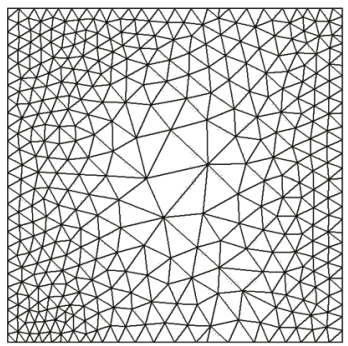

(c) Mesh independent (MLS)

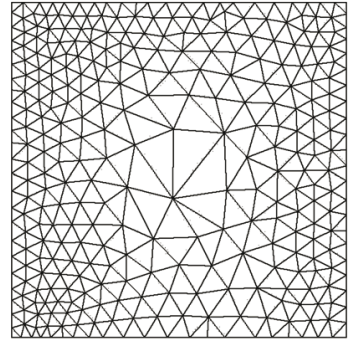

(d) Mesh Based (MLS)

Fig. 9 Adaptively refined mesh in plate problem domain using displacement recovery techniques [quadratic initial mesh elements $=45,0.1 \%$ target error, $\mathrm{L}_{2}$ norm]

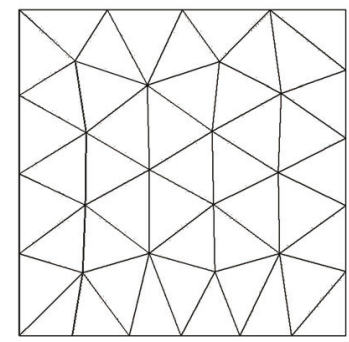

(a) Initial Mesh

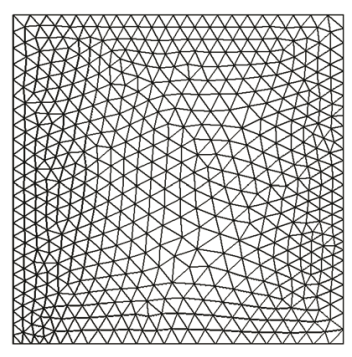

(b) Mesh Based (LS)

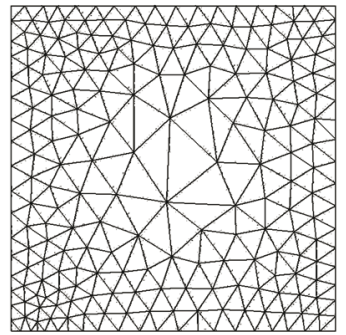

(c) Mesh Independent (MLS)

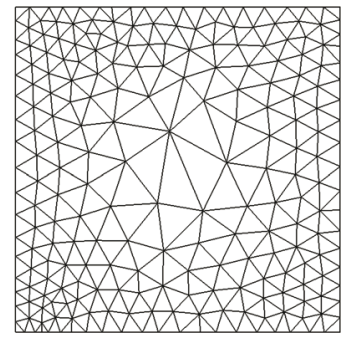

(d) Mesh Based (MLS)

Fig. 10 Adaptively refined mesh in plate problem domain using displacement recovery techniques [quadratic initial mesh elements $=45,1 \%$ target error, energy norm] 


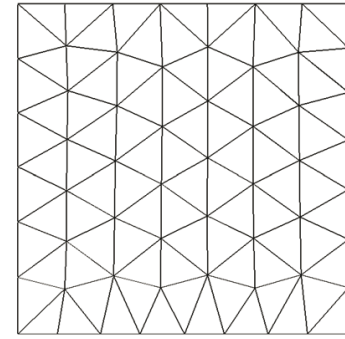

(a) Initial mesh

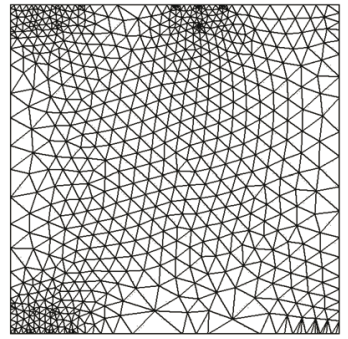

(b) Mesh based (LS)

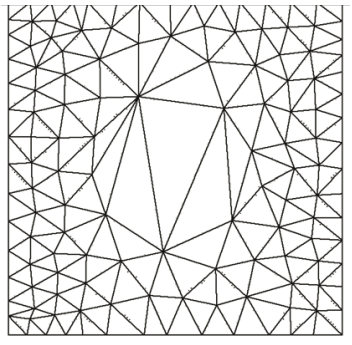

(c) Mesh independent (MLS)

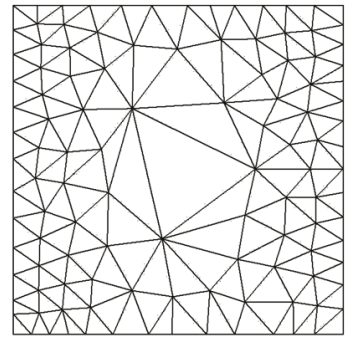

(d) Mesh based (MLS)

Fig. 11 Adaptively refined meshes in plate problem domain using displacement recovery techniques [quadratic initial mesh elements $=88,0.1 \%$ target error, $\mathrm{L}_{2}$ norm]

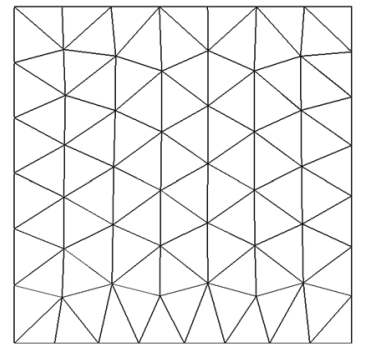

(a) Initial mesh

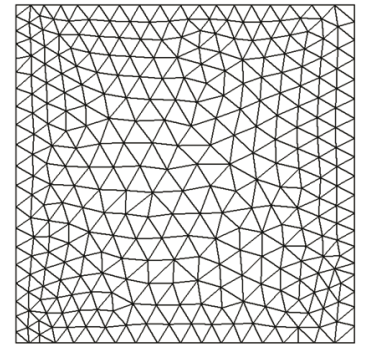

(b) Mesh based (LS)

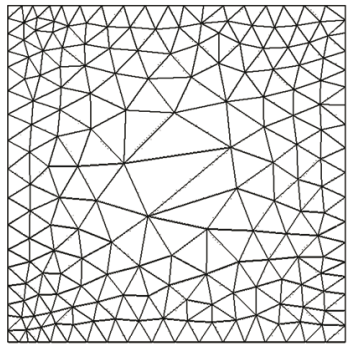

(c) Mesh independent (MLS)

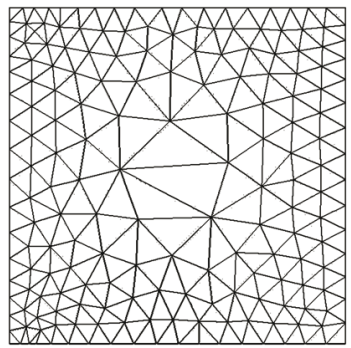

(d) Mesh based (MLS)

Fig. 12 Adaptively refined meshes in plate problem domain using displacement recovery techniques [quadratic initial mesh elements $=88,1 \%$ target error, energy norm]

\section{Conclusions}

In the present study, techniques using mesh independent and mesh dependent moving least square interpolation for displacement recovery of the finite element solution discretization error quantified in both the $\mathrm{L}_{2}$ and the energy norm are proposed, and the performance of the recovery techniques is demonstrated on benchmark elastic plate problems. The mesh independent recovery procedure is based on the recovery of displacement over a patch of nodes, independent of mesh connectivity, in the form of circular zones of the discretized domain. The derivatives at any point in the element domain or node domain are calculated from a better approximated displacement field using the least square fit of the computed nodal displacement considering a higher order polynomial over a mesh dependent element patch. The derivatives are also calculated using the moving weighted least square fit of the computed nodal displacement considering a higher order polynomial over a patch of mesh independent (or mesh dependent) nodes. The approach is improved with respect to the existing approaches in which the derivatives are calculated from nodal values of stresses using the same order polynomial function as that present in the basis function valid over an element patch surrounding the assembly node considered.

The performance of the error estimator based on the mesh independent displacement recovery has been compared with that of the error estimator based on the mesh dependent displacement recovery, using the moving least square and least square interpolation techniques. It is found that the MLS based recovery scheme results in more accurate displacement and displacement gradients than the least square displacement recovery scheme. The error convergence obtained by using the mesh independent and mesh dependent MLS based recovery schemes is found to be better than the least square displacement recovery scheme under the energy and the L2 norm for the error measure. In addition, the order of error in the MLS based recovery scheme is smaller in comparison to the least square displacement recovery scheme, hence the MLS recovery scheme is more effective than the least square recovery scheme. The numerical results also show that the $\mathrm{L}_{2}$ norm for error representation should be used with higher order elements for optimal performance. It is concluded that the 
improved quality of error estimation is obtained by using the MLS interpolation technique instead of the least square interpolation technique. The error computations using the mesh based/mesh independent displacement recovery can be implemented in an adaptive analysis to predict high error zones and to control the solution error effectively. The finite element analysis based on the mesh dependent MLS displacement recovery is very effective in converging to a predefined accuracy in the solution with a significantly smaller number of degrees of freedom in comparison to the mesh independent MLS displacement recovery and the analysis based on the mesh dependent least square displacement recovery.

\section{Acknowledgements and funding}

The author expresses his appreciation to the Deanship of Scientific Research at King Khalid University for funding this study through the General Research Project under grant number [R.G.P2 /73/41]. The author gratefully acknowledges the valuable support and help of the Dean of the Faculty of Engineering.

\section{REFERENCES}

[1] Chen, J.-S., Hillman, M. M., Chi, S-W., Mesh free Methods: Progress Made after 20 Years, J. Eng. Mechanics, 143-4 (2017) 1-38. https://doi.org/10.1061/(asce)em.1943-7889.0001176

[2] Kim, J., Bathe, Klaus-J., Towards a procedure to automatically improve finite element solutions by interpolation covers, Computers and Structures, 131-150 (2014) 81-97. https://doi.org/10.1016/j.compstruc.2013.09.007

[3] Li, W., Thanh, N. N., Huanga,J., Zhoua, K., Adaptive analysis of crack propagation in thin-shell structures via an isogeometric-mesh free moving least-squares approach, Comput. Methods Appl. Mech. Engrg. 2020. https://doi.org/10.1016/j.cma.2019.112613

[4] Cortis, M., Coombs, W., Augarde, C., Brown, M., Brennan, A., Robinson, S., Imposition of essential boundary conditions in the material point method, Int. J. Num. Meth. Engg., 113-1 (2018) 130-152. https://doi.org/10.1002/nme.5606

[5] Liu, Y., Liu, Y., Ding, K., A new coupling technique for the combination of wavelet Galerkin method with finite element method in solids and structures, Int. J. Num. Meth. Engg., 112-10 (2017) 1295-1322. https://doi.org/10.1002/nme.5558

[6] Dekker, R., van der Meer, F.P., Maljaars, J., Sluys, L.J., A cohesive XFEM model for simulating fatigue crack growth under mixed-mode loading and overloading, Int. J. Num. Meth. Engg., (2019). https://doi.org/10.1002/nme.6026

[7] Igelbüscher, M., Schwarz, A., Steeger, K., Schröder, J., Modified mixed least-squares finite element formulations for small and finite strain plasticity, Int. J. Num. Meth. Engg., 117-1 (2019) 141-160. https://doi.org/10.1002/nme.5951

[8] Lee, C.-Kye, Mihai, L. A., Hale, J. S., Kerfriden, P., Bordas, S. P.A., Strain smoothing for compressible and nearly-incompressible finite elasticity, Computers and Structures, 182 (2017) 540-555. https://doi.org/10.1016/j.compstruc.2016.05.004

[9] Jarak, T., Jalušić, B., Sorić, J., Mixed Meshless Local Petrov-Galerkin Methods For Solving Linear Fourth-Order Differential Equations, Transactions of Famena, 2020. https://doi.org/10.21278/TOF.44101

[10] Gratsch, T., Bathe, K., A Posteriori error estimation techniques in practical finite element analysis, Computers and Structures, 83 (2005) 75-90. https://doi.org/10.1016/j.compstruc.2004.08.011

[11] Zienkiewicz O. C., Zhu J. Z., Simple error estimator and adaptive procedure for practical engineering analysis, Int J. Numer. Meth. Engg. 24 (1987) 337-357. https://doi.org/10.1002/nme.1620240206

[12] Li, X. D., Wiberg, N. E., A posteriori error estimate by element patch post-processing, adaptive analysis in Energy and $\mathrm{L}_{2}$ Norms, Comp. and Structures, 53-4 (1994) 907-919. https://doi.org/10.1016/00457949(94)90378-6

[13] Grosse, I.R., Katragadda, P., Benoit, J., An adaptive accuracy-based a posteriori error estimator, J. Finite Elem. Anal. Design, 12-1 (1994) 831-841. https://doi.org/10.1016/0168-874x(92)90008-z

[14] Ahmed M., Singh D., An Adaptive Parametric Study on Mesh Refinement during Adaptive Finite Element Simulation of Sheet Forming Operations, Turkish J. Eng. Env. Science, 32 (2008) 163-175.

[15] Zienkiewicz O. C., Zhu J. Z., The Super-convergent Patch Recovery and a posteriori Error Estimates, Part I, The Error Recovery Technique, Int. J. Num. Meth. Engg., 33 (1992) 1331-1364. https://doi.org/10.1002/nme.1620330702 
[16] Ahmed, M., Singh, D., Islam, S., Effect of Contact Conditions on Adaptive Finite Element Simulation of Sheet Forming Operations, European Journal of Computational Mechanics, 24-1 (2015) 1-15. https://doi.org/10.1080/17797179.2015.1012632

[17] Chen, J., Chen, Z., Three-dimensional super-convergent gradient recovery on tetrahedral meshes, Int J. Numer. Meth. Engg., 108-8 (2016) 819-838. https://doi.org/10.1002/nme.5229

[18] Zhang, R., Li, L., Zhao, L., Tang, G., An adaptive remeshing procedure for discontinuous finite element limit analysis, Int J. Numer. Meth. Engg., 118-5 (2018) 287-307. https://doi.org/10.1002/nme.5925

[19] Gavete, L., Falcón, S., Ruiz, A., An error indicator for the element free Galerkin method, European J. Mech., A/Solids, 20 (2001) 327-341. https://doi.org/10.1016/s0997-7538(00)01132-3

[20] Kim, K., Lee, H., A posteriori Error Estimator for non-conforming finite element methods of the linear elasticity problem, Journal of Computational and Applied Mathematics, 235 (2010) 186-202. https://doi.org/10.1016/j.cam.2010.05.032

[21] Nadal, E., D'1ez, P., R'odenas, J.J., Tur, M., Fuenmayor, F.J., A recovery-explicit error estimator in energy norm for linear elasticity, Computer Methods in Applied Mechanics and Engineering, 287 (2015) 172-190. https://doi.org/10.1016/j.cma.2015.01.013

[22] Abbas, A., Abdul Rahman, R., Adaptive FEM with Domain Decomposition Method for PartitionedBased Fluid-Structure Interaction, Arabian Journal Science \& Engg., 41-2 (2016) 611-622. https://doi.org/10.1007/s13369-015-1708-8

[23] Ülkü, H., Karaköse, C., Askes, H., A recovery-type a posteriori error estimator for gradient elasticity, Computers and Structures, 154 (2015) 204-209. https://doi.org/10.1016/j.compstruc.2015.04.003

[24] Yu, T., Bui, T. Q., Numerical simulation of 2-D weak and strong discontinuities by a novel approach based on XFEM with local mesh refinement, Computers and Structures, 196 (2018) 112-133. https://doi.org/10.1016/j.compstruc.2017.11.007

[25] Bird, R.E., Giani, S., Coombs, W.M., A posteriori discontinuous Galerkin error estimator for linear elasticity, Applied Mathematics and Computation, 2019. https://doi.org/10.1016/j.amc.2018.08.039

[26] Mirzaei, D., Analysis of moving least squares approximation revisited", J. Computational and Applied Mathematics, 282 (2015) 237-250. https://doi.org/10.1016/j.cam.2015.01.007

[27] Ullah, Z., Coombs, W.M., Augarde, C.E., An adaptive finite element/meshless coupled method based on local maximum entropy shape functions for linear and nonlinear problems, Computer Methods in Applied Mechanics and Engineering, 267 (2013) 111-132. https://doi.org/10.1016/j.cma.2013.07.018

[28] Parret-Fréaud, A., Rey, V., Gosselet, P., Rey, C., Improved recovery of admissible stress in domain decomposition methods- Application to heterogeneous structures and new error bounds for FETI-DP, Int. Journal for Numerical Methods in Engg., 111-1 (2016) 69-87. https://doi.org/10.1002/nme.5462

[29] Chen, J.S., Yoon, S., Wu, C.T., Non-linear version of stabilized conforming nodal integration for Galerkin mesh-free methods. Int J Numer Methods Engg., 53 (2002) 2587-2615. https://doi.org/10.1002/nme.338

[30] Chung, H-J., Belytschko, T., An error estimate in the EFG method. Computational Mechanics, 21 (1998) 91-100. https://doi.org/10.1007/s004660050286

[31] Lee CK, Zhou CE., On error estimation and adaptive refinement for element free Galerkin method: Part I: stress recovery and a posteriori error estimation. Comput Structure, 82-4/5 (2004) 413-428. https://doi.org/10.1016/j.compstruc.2003.10.018

[32] He, Y., Yang, H., Deeks. A. J., A node-based error estimator for the element-free Galerkin (EFG) Method. August 2014 International Journal of Computational Methods, 11-04 (2014) 1350059. https://doi.org/10.1142/s021987621350059x

[33] Ahmed, M., Singh, D., Desmukh, M.N., Interpolation Type Stress Recovery Technique Based Error Estimator for Elasticity Problems. Mechanics, 24-5 (2018) 672-679. https://doi.org/10.5755/j01.mech.24.5.19937

[34] Onate E., Perazzo, F., Miquel, J., A finite point method for elasticity problems, Computers and Structures, 79 (2001) 2151-2163. https://doi.org/10.1016/s0045-7949(01)00067-0

Submitted: $\quad 22.6 .2020$

Accepted: $\quad 30.3 .3021$
Mohd Ahmed*

Civil Engineering Department, College of Engineering, King Khalid University, Abha-61421, Saudi Arabia

*Correspondence: mall@kku.edu.sa 